\title{
Research Paper: Comparing the Isometric Strength of the Shoulder and Scapulothoracic Muscles in Vol- leyball and Futsal Athletes
}

\author{
Afshin Rasouli ${ }^{1}$, Aliashraf Jamshidi ${ }^{*}$, Soheil Sohani ${ }^{1}$
}

1. Department of Physiotherapy, School of Rehabilitation, Iran University of Medical Sciences, Tehran, Iran.

Crtation: Rasouli A, Jamshidi A, Sohani S. Comparing the Isometric Strength of the Shoulder and Scapulothoracic Muscles in Volleyball and Futsal Athletes. Physical Treatments. 2017; 7(1):41-48. https://doi.org/10.29252/nrip.ptj.7.1.41

Article info:

Received: 20 Oct. 2016

Accepted: 04 Feb. 2017
Keywords:

Overhead athletes, Shoulder, Strength ratio, Scapulothoracic

\begin{abstract}
A B S T RA C T
Purpose: This study aimed to evaluate the symmetry of the isometric strength of the shoulder and scapulothoracic muscles in volleyball and futsal athletes.

Methods: In this case-control study, the strength of the scapulothoracic muscles and rotation of the shoulders in 20 adult volleyball athletes and 20 futsal adult athletes, who were randomly selected from sports clubs around Tehran, were measured using a manual dynamometer. The obtained data between the different groups and within the individual groups were compared by Independent and paired $t$ tests, respectively.

Results: The isometric strength of the external rotator muscles, internal rotator muscles, upper trapezius and serratus anterior of the dominant side of volleyball and futsal athletes were different $(\mathrm{P}<0.05)$. The isometric strength of the same muscles in the dominant side of the volleyball athletes was more than the non-dominant side $(\mathrm{P}<0.05)$. However, the isometric strength of the middle trapezius and rhomboid muscles were less. The ratio of agonist to antagonist, in all muscle groups between two groups, in the dominant side as well as between the dominant and non-dominant side, were different in volleyball athletes.

Conclusion: In volleyball athletes, disturbances in the strength ratio of the agonist and antagonist muscles of the scapula, and weaknesses in these muscles, impair the dynamic stability of the scapula and shoulder joints and disturbances in the rhythm of the scapulohumeral muscles. It is recommended that during training, assessment and treatment of these athletes strengthening these muscles be considered.
\end{abstract}

\section{Introduction}

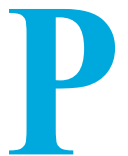

revalence of shoulder pain ranks the second among musculoskeletal pains and affects $20 \%$ to $22 \%$ of general population [1]. Disorders of the stabilizing muscles of the scapula can be an important factor in abnormal biomechanics and lesions of the shoulder girdle [2]. Static shoulder joint is stable when the arms are positioned at the side of the body by the capsuloligamentous components. However, when the arms move, rotator cuff muscles are responsible for dynamic stability [3]. The

\section{* Corresponding Author:}

Aliashraf Jamshidi, PhD

Address: Department of Physiotherapy, School of Rehabilitation, Iran University of Medical Sciences, Tehran, Iran.

Phone: +98 (912) 0304315

E-mail: afshinrasoulifasih@yahoo.com 
control of shoulder complex movements are performed by muscles in a matched manner [3].

In repetitive overhead throwing athletes, dynamic stability is important [4]. Traditional overhead sports are handball, baseball, volleyball, and basketball [4]. Shoulder injuries are common in overhead sport athletes [5, $6]$. In various studies, shoulder problems are reported as $44 \%$ and shoulder pain as 29\% [6]. Eccentric contraction imposes excessive load on rotator cuff muscle. Injury is induced by muscle imbalance and eventually shoulder instability or shoulder impingement syndrome will occur [4]. In throwing and serving, scapula facilitates shoulder movements and provides suitable positioning. Research in glenohumeral abnormalities has shown that serratus anterior, rhomboids, middle and lower trapezius are weak or inhibited [7]. In the impingement syndrome, there are decreased scapular upward rotation, increased anterior tipping, and increased scapular medial rotation [8]. In baseball athletes, there was an increase in external rotation range and a decrease in muscle strength, particularly the supraspinatus muscles [5].

In handball athletes, the range of motion of the shoulders remains unchanged but the strength of the rotational muscles increases [9]. Athletes competing in overhead sports, who besides shoulder impingement syndrome developed drooping shoulders, reported an increase in the upper trapezius muscle strength during abduction, external rotation and a decrease in median trapezius functionality during abduction and lower trapezium during external rotation [10]. Disturbances in scapular movement can cause problems such as abnormal tension around the anterior capsule of the shoulder joint, higher tension in rotator cuff muscles, and a decrease in muscle activity $[7,11]$.

Unfortunately, most studies have done on specific muscle groups. In these studies, the scapulothoracic joint, which plays an important role in scapulohumeral rhythm, have been studied less extensively. Dysfunction of these muscles cause instability and eventually disturbances in the normal rhythm of the scapulothoracic joint. Thus, evaluation of the scapulothoracic muscles and rotation of the shoulder and its symmetry should be investigated further. This study aimed to compare the ratio of the shoulder muscles strength symmetry and scapulothoracic joint in professional volleyball and futsal players.

\section{Materials and Methods}

The present study was a case-control study. Testing was performed at the sports clubs of Tehran in 2017. A total of 40 participants (20 volleyball and 20 futsal play- ers) at the club level were selected with simple random sampling method. Then the study samples were divided into two groups. The subjects were adult male volleyball athletes from various clubs in Tehran, with three years of experience doing overhead sports. They regularly played overhead sports at least three times a week [12].

The non-overhead group consisted of futsal athletes. The participants were between 17 and 30 years old and did not have a history of systemic disease, rheumatism, neurological disease, fractures, dislocations, or surgery of the shoulder joints. The selection criteria for the healthy participants included age compliance, gender, body mass index matching with athletic group, absence of shoulder pain, surgery and any shoulder pathology. Before data collection, the subjects read and signed informed consent forms approved by the Iran University of Medical Sciences Ethics Committee (IR.IUMS. REC1395.9311340004). Muscle strength was measured by a handheld dynamometer (Imada design) with the ability to measure tensional strengths and a pressure capacity of $500 \mathrm{~N}$, and a precision of $0.1 \mathrm{~N}$ (Figure 1).

The subjects were asked to keep a maximum voluntary contraction for 5 seconds. Each contraction was repeated three times with 30 seconds rest between each try [13-15]. In this study, the muscles are not usually tested individually and other muscles are involved [15]. For example, various studies have shown increased activity of the medial trapezius muscle during the lower trape-

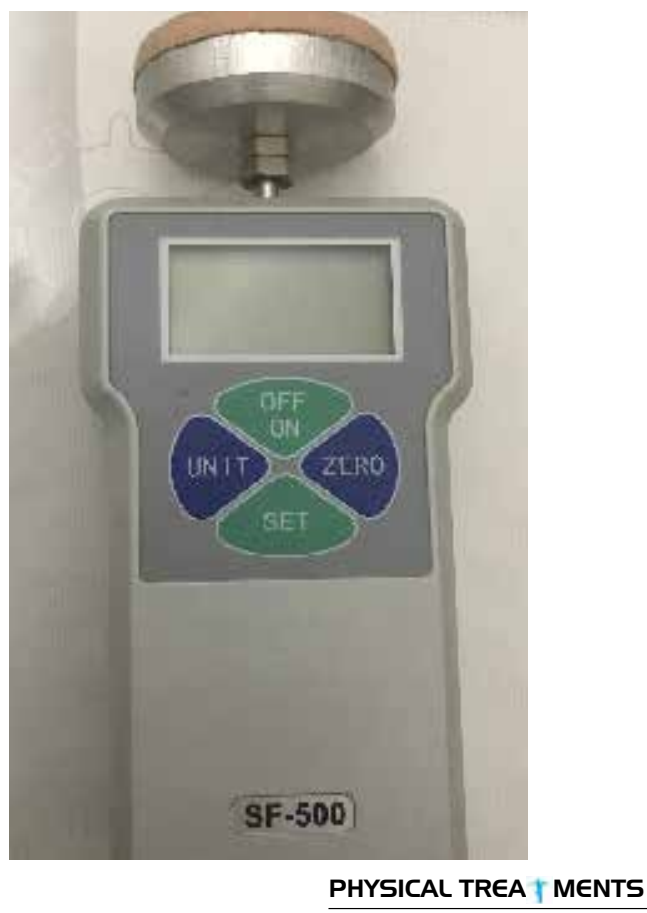

Figure 1. Imada manual dynamometer 
zius test [16-18]. To measure the maximum isometric strength of lower trapezius, the subject was asked to lie down and position his shoulder in 145 degrees abduction along with external rotation. The experimenter, using one hand, would position the scapula on the opposite side and place the handheld dynamometer on the external side of the acromion of the side being tested, and then applied resistance against scapula adduction and depression $[15,17,19]$.

To measure the maximum isometric strength of the serratus anterior, the subject was asked to sit, flex his 130 degrees and place his scapula in an abduction position. The dynamometer was placed on the humerus bone and the distal deltoid muscle until protraction and rotation of the upper scapula took place simultaneously $[16,18,20]$. To measure the maximum isometric strength of the upper trapezius, the subject was asked to sit, the dynamometer was placed in the region of the acromion and clavicle, and pressure was applied in a downward direction, and at the same time the subject was asked to elevate his scapula $[13,17,19]$ (Figure 2). To measure the maximum isometric strength of the medial trapezius, while the subject was lying in a prone position, the shoulder was bent 90 degrees abduction and the elbow was bent 90 degrees, and the handheld dynamometer was positioned on the external rare acromion. At the same time the subject had to bring their scapula closer together and then retract them. The scapula in this state has to be retracted $[15,17,19]$.

To measure the maximum isometric strength of the rhomboid muscles, the subject was sat in a prone position, place the limb that was being tested around their waist, along with internal rotation of the shoulder and abduction and downward rotation of the scapula, without elevation, and the handheld dynamometer was placed between the external epicondyle and acromion. As the subject was applying strength in the direction of adduction using their upper limb, the examiner would exert pressure on the abduction side [19,21].

To measure the maximum isometric strength of the external and internal rotators, the subject was placed in a prone position, bent his elbow 90 degrees, place the shoulders in a 90 degrees abduction and hang the limbs off from the bed, with a folded towel under the shoulder. The handheld dynamometer was placed in the dorsal surface of the hand (above the wrist joint) for external rotation and in the anterior surface of the hand (above the wrist joint) for internal rotation. The subject applied pressure to the external rotation and the examiner towards the internal. The subject was asked to apply maximum pressure on the dynamometer in the directions of

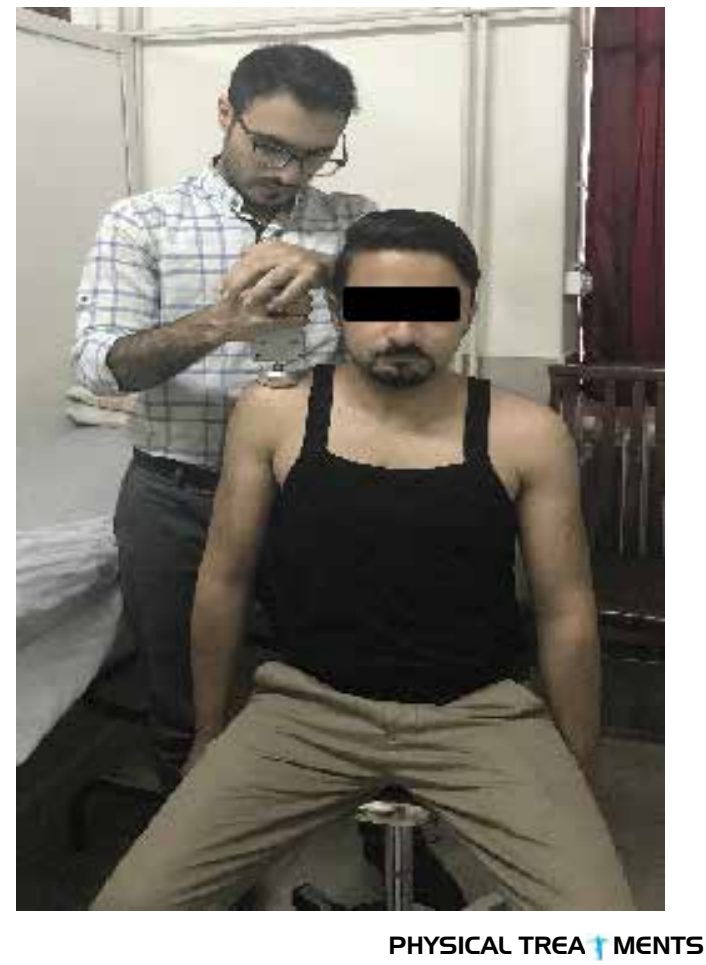

Figure 2. Upper trapezius manual muscle test. The subject was seated with his arms at the sides. The dynamometer was placed halfway between the mastoid and lateral acromion over the muscle bulk. The examiner was allowed to use both hands

the external rotation as well as the internal rotation that was held by the examiner [19, 22].

Muscle weakness and muscle imbalance can cause impingement of the shoulder joint syndrome, therefore, evaluation of the ratio of antagonist-agonist of the scapulothoracic muscle on one side can help diagnose and treat $[2,8,22-24]$. To measure the elevation to depression ratio of the scapula, the upper trapezius muscle was considered for elevation and the lower trapezius for depression [15].

To evaluate the relationship between upward and downward rotations of the scapula, the serratus anterior muscle was considered for upward rotation of the scapula and the rhomboid muscles for its downward rotation. For the sake of simplicity, instead of using the powercoupled strength of the upper and lower trapezius muscles and serratus anterior for the rotation of the upward scapula, only the serratus anterior muscle was used [15].

To evaluate the relationship between scapular protraction and retraction, the serratus anterior muscle was considered for protraction and the medial trapezius muscle for retraction. The upper trapezius muscle was chosen because its only function is retraction in contrast to the 
rhomboids, which have retraction and downward rotation functional abilities [15]. To evaluate the ratio of external to internal rotation, for external rotation of the muscle, the external rotator of the shoulder and for internal rotation the internal rotator muscle of the shoulder were considered.

\section{Statistical analyses}

Descriptive statistics were calculated for all variables, and all dependent variables were controlled for normal distribution of variance using the 1-sample KolmogorovSmirnov test. As all data were normally distributed with equal variances, independent $t$ test was used to compare the isometric strength of shoulder muscle in two groups of futsal and volleyball, and the paired t test was used to compare the left and right sides in two groups. The level of significance for all the tests was considered to be $<0.05$. Data were analyzed using SPSS, version 18 .

\section{Ethical considerations}

After visiting the test setting, the participants signed informed consent forms approved by the Ethics Committee of Iran University of Medical Sciences.

\section{Results}

The demographics of the volleyball group were as follows: mean(SD) age; 22.75(2.48) years, mean(SD) Body Mass Index (BMI); 20.27(5.08) kg/m², mean(SD) history of sports activity; 62.30(18.22) months, mean(SD) number of practice sessions per week; 3.05(22.00). The futsal group demographics were as follows: mean(SD) age; 23.70(1.62), mean(SD) BMI; 20.592(7.00) kg/ $\mathrm{m}^{2}$, mean(SD) history of sports activity; 58.15(20.27) months, mean(SD) number of practice sessions per week 3.25(55.00). There was no statistically significant difference between two groups (Table 1).

The maximum isometric muscle strength of internal and external rotator muscles, upper trapezius muscle, and serratus anterior of the dominant side were statistically significant in both groups. Whereas, the maximum strength of the middle and lower trapezius muscle, and the rhomboid muscles were not statistically significant (Table 2). The maximum isometric strength, between the dominant and non-dominant side, of the external and internal rotator muscles, serratus anterior, upper and middle trapezius, and rhomboids was statically significant in the volleyball group. The maximum isometric strength of the lower trapezius, between the dominant and nondominant of the volleyball group, were not statically significant (Table 3).

The ratios of the maximum strength of the external rotation to the internal rotation, upward rotation to the downward rotation, protraction to retraction, and elevation to depression in the dominant side, between the groups, were statistically significant (Figure 3 ). The ratios of the maximum strength of the external rotation to the internal rotation, upward rotation to the downward rotation, protraction to retraction, and elevation to depression, between the dominant and non-dominant side, in the volleyball group and in dominate side between two groups, were statistically significant (Figure 3 and 4).

\section{Discussion}

The strength of internal and external rotator muscles of the dominant shoulder in volleyball athletes was more than that in futsal athletes. These findings were consistent with those of Barlow and Alfredson [12, 25]. These changes result from the type of the exercise they perform. Research has shown that one-side sports such as volleyball causes accommodative changes in muscle strength within the dominant side [26]. The strength of the upper trapezius muscle of the dominant side in volleyball athletes was more than that in futsal athletes, which was in accordance with the findings of Ludewig [8]. In volleyball players, during the cocking and acceleration phases, the upper trapezius and serratus anterior play a significant role throughout the course of throwing

Table 1. Demographic characteristics of the studied population

\begin{tabular}{cccc}
\hline & Futsal $(\mathbf{n}=\mathbf{2 0})$ Mean \pm SD & Volleyball $(\mathbf{n = 2 0})$ Mean \pm SD & P \\
\hline Age $(\mathrm{y})$ & $23.70 \pm 1.62$ & $22.75 \pm 2.48$ & 0.1445 \\
BMl $\left(\mathrm{kg} / \mathrm{m}^{2}\right)$ & $20.592 \pm 7.00$ & $20.27 \pm 5.08$ & 0.728 \\
Sports experience $(\mathrm{mon})$ & $58.15 \pm 20.27$ & $62.30 \pm 18.22$ & 0.5001 \\
Number of sessions & $3.25 \pm 55.00$ & $3.05 \pm 22.00$ & 0.1625 \\
\hline
\end{tabular}


Table 2. Independent $t$ test comparing the dominant muscle strength between two groups

\begin{tabular}{|c|c|c|c|}
\hline Muscle & Futsal Players $(n=20)$ Mean $\pm S D$ & Volleyball Players $(n=20)$ Mean $\pm S D$ & $\mathbf{P}$ \\
\hline External rotators & $11.92 \pm 1.70$ & $15.55 \pm 1.13$ & $* 0.001$ \\
\hline Internal rotators & $13.68 \pm 2.29$ & $18.78 \pm 1.76$ & $* 0.001$ \\
\hline Upper trapezius & $28.25 \pm 3.20$ & $31.85 \pm 2.03$ & $* 0.001$ \\
\hline Middle trapezius & $26.67 \pm 3.12$ & $25.76 \pm 0.91$ & 0.224 \\
\hline Lower trapezius & $24.07 \pm 2.95$ & $22.78 \pm 1.15$ & 0.077 \\
\hline Serratus anterior & $24.71 \pm 3.71$ & $30.92 \pm 2.45$ & $*^{*} 0.001$ \\
\hline Rhomboids & $16.26 \pm 1.58$ & $15.45 \pm 0.90$ & 0.057 \\
\hline
\end{tabular}

* Indicates the significant difference.

movements that places the scapula in an elevation and upper rotation position [27].

The strength of the upper trapezius muscle, serratus anterior and rhomboids of the dominant side, in the volleyball group, in comparison with the dominant side of the futsal group, did not show a statistically significant difference. This was consistent with the findings of Cools and associates [10]. Research has shown that the medial trapezius, anterior trapezius and rhomboid muscles in athletes with overhead activities, does not affect the rest of their muscles [27]. The strength of the external and internal rotator muscles and the upper trapezius and serratus anterior of the dominant side, compared to the nondominant side, was more in both groups of athletes. The findings of Turner, Wilk, and Myers all support these results $[15,26,28]$. The reason for this difference can be attributed to neural adaptation of the muscle on the dominant side [29].

The strength of the middle trapezius muscle and rhomboids of the dominant hand, compared to the non-domi- nant side, was less in volleyball players. This was consistent with the results obtained by Cools, too [10]. These muscles control the speed of the scapula movements eccentrically. Research has shown weakness of these muscles in athletes suffering from shoulder impingement. Given that shoulder impingement is common in volleyball players, weakness of their medial trapezius and rhomboids muscles could result in hypermobility of the scapula. Following the weakness of these muscle, the rhythm of the scapulohumeral is disturbed and the risk of functional impingement of the shoulders may increase $[3,30]$.

The strength of the lower trapezius muscle of the dominant side, compared to the non-dominant side, in volleyball athletes did not show a statistically significant difference. These results were consistent with that of Cools and Barlow et al. [10, 12]. Research has shown that lower trapezius muscle compared to other muscles plays no role in overhead activities [27].

The ratio of the external rotator muscle to internal rotator one, of the dominant side, in the volleyball group was

Table 3. Paired $t$ test to compare dominant and non-dominant muscle strength within volleyball group $(n=20)$

\begin{tabular}{|c|c|c|c|}
\hline Muscle & Dominant Mean $\pm S D$ & Non-dominant Mean \pm SD & $\mathbf{P}$ \\
\hline External rotators & $15.16 \pm 1.10$ & $15.55 \pm 1.13$ & $* 0.020$ \\
\hline Internal rotators & $16.44 \pm 1.56$ & $18.78 \pm 1.76$ & $* 0.001$ \\
\hline Upper trapezius & $28.71 \pm 3.26$ & $31.85 \pm 2.03$ & $* 0.001$ \\
\hline Middle trapezius & $27.82 \pm 1.29$ & $25.76 \pm .91$ & $* 0.001$ \\
\hline Lower trapezius & $22.30 \pm 1.30$ & $22.07 \pm 1.15$ & 0.086 \\
\hline Serratus anterior & $28.05 \pm 2.15$ & $30.92 \pm 2.45$ & $* 0.001$ \\
\hline Rhomboids & $16.49 \pm .87$ & $15.45 \pm .90$ & $* 0.001$ \\
\hline
\end{tabular}

* Indicates the significant difference. 


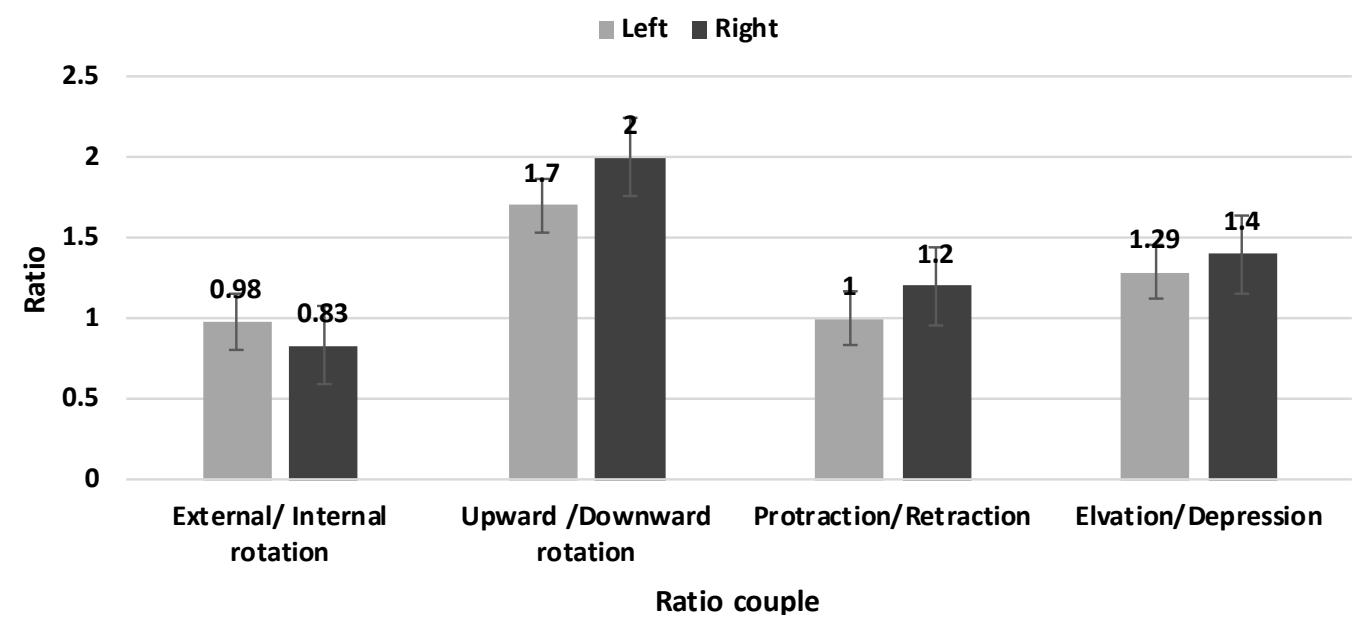

PHYSICAL TREA MENTS

Figure 3. Independent $t$ test comparing the strength ratio of agonist to antagonist muscles in dominant side between two groups

* Indicates the significant difference

less compared to the futsal group. Moreover, the ratio of the external to internal rotator muscle strength of volleyball athletes was less in the dominant side compared to the non-dominant side. These results were consistent with the findings of Donatelli [31]. Research supports that external rotator muscles are less active than internal rotator muscles in overhead athletes [27].

The ratio of the strength of the dominant upward rotator scapula to the downward rotator in the volleyball group was more than that ratio in the futsal group. Furthermore, this ratio in volleyball athletes was more in the dominant arm than the non-dominant arm. The findings of Turner and colleagues also showed that in the dominant side, the ratio of the upper scapula rotator to the lower rotator was more than that in the non-dominant side [15].

The ratio of protraction to retraction of the scapula of the volleyball group in the dominant side was more than that of the futsal group. Moreover, this ratio in volleyball athletes was more in the dominant hand than in the non-dominant one, which was consistent with the findings of Cools and colleagues [10]. Table 2 demonstrates that disturbances in this ratio is caused by an increase in strength of the serratus anterior and a decrease in the strength of the middle trapezius muscles and rhomboids of the dominant hand, compared to the non-dominant side, in volleyball players.

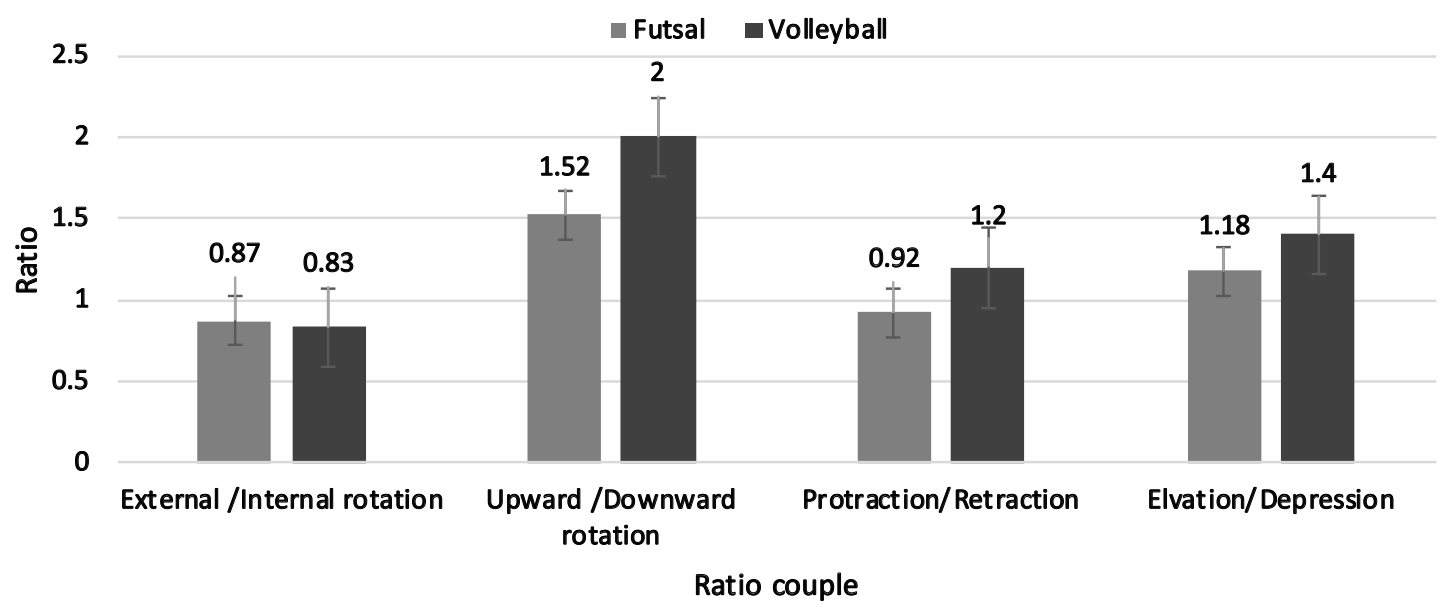

Figure 4. Paired $t$ test comparing the strength ratio of right and left side within volleyball group

PHYSICAL TREA MENTS

* Indicates the significant difference 
Defects in the strength of scapula retraction decrease the stability in the cocking movement of athletes [7]. An increase in protraction causes impingement and shortness of the shoulder's joint capsule [7]. These findings explain the increased risk of injury in overhead athletes. The ratio of elevation to depression strength of the dominant hand was more in the volleyball group in comparison with the futsal group. Furthermore, in the volleyball group, the ratio of agonist to antagonist elevation to depression of the dominant hand was more compared to the non-dominant hand. These results were consistent with the findings of Cools and Turner [10, 15].

Weakness of medial trapezius and rhomboid in volleyball players that results in hypermobility in the scapula, decrease the speed of movement in the scapula, as well as the ratio in volleyball athletes. Inappropriate strength ratio of the agonist and antagonist muscles of the scapula, and weaknesses in these muscles, impairs the dynamic stability of the scapula and shoulder joints and disturbances in scapulohumeral rhythm. Defects in the strength of scapula retraction decrease the stability in the cocking movement of athletes. An increase in protraction causes impingement and shortness of the shoulder's joint capsule. These findings explain the increased risk of injury in overhead athletes. Thus, the balance of power in these muscles must be regarded during the training programs of these athletes. In future studies the strength of these muscles could be examined in the form of eccentric and concentric manner.

\section{Acknowledgements}

This study was extracted from Rasouli Fasih's master thesis at the School of Rehabilitation, Iran University of Medical Sciences. Financially, it was supported by Iran University of Medical Sciences.

\section{Conflict of Interest}

The authors declared no conflicts of interest.

\section{References}

[1] Picavet HSJ, Schouten JSAG. Musculoskeletal pain in the Netherlands: Prevalences, consequences and risk groups, the DMC3-study. Pain. 2003; 102(1):167-78. doi: 10.1016/ s0304-3959(02)00372-x

[2] Kamkar A, Irrgang JJ, Whitney SL. Nonoperative Management of secondary shoulder impingement syndrome.
Journal of Orthopaedic \& Sports Physical Therapy. 1993; 17(5):212-24. doi: 10.2519/jospt.1993.17.5.212

[3] Hertling, D, Kessler RM. Management of common musculoskeletal disorders: Physical therapy principles and methods. Philadelphia: Lippincott Williams \& Wilkins; 2006.

[4] Baltaci G, Tunay VB. Isokinetic performance at diagonal pattern and shoulder mobility in elite overhead athletes. Scandinavian Journal of Medicine and Science in Sports. 2004; 14(4):231-8. doi: 10.1111/j.1600-0838.2004.00348.x

[5] Magnusson, SP, Gleim GW, Nicholas JA. Shoulder weakness in professional baseball pitchers. Medicine \& Science in Sports \& Exercise. 1994; 26(1):5-9. doi: 10.1249/00005768199401000-00003

[6] Lo YP, Hsu YC, Chan KM. Epidemiology of shoulder impingement in upper arm sports events. British Journal of Sports Medicine. BMJ; 1990; 24(3):173-7. doi: 10.1136/ bjsm.24.3.173

[7] Ben Kibler W. The Role of the Scapula in Athletic Shoulder Function. American Journal of Sports Medicine. 1998; 26(2):325-37. doi: 10.1177/03635465980260022801

[8] Ludewig PM, Cook TM. Alterations in shoulder kinematics and associated muscle activity in people with symptoms of shoulder impingement. Physical Therapy. 2000; 80(3):27691. doi: $10.1093 / \mathrm{ptj} / 80.3 .276$

[9] Fieseler G, Jungermann P, Koke A, Irlenbusch L, Delank KS, Schwesig R. Range of motion and isometric strength of shoulder joints of team handball athletes during the playing season, part II: Changes after midseason. Journal of Shoulder and Elbow Surgery. 2015; 24(3):391-8. doi: 10.1016/j. jse.2014.07.019

[10] Cools AM, Declercq GA, Cambier DC, Mahieu NN, Witvrouw EE. Trapezius activity and intramuscular balance during isokinetic exercise in overhead athletes with impingement symptoms. Scandinavian Journal of Medicine and Science in Sports. 2006; 17(1):25-33. doi: 10.1111/j.16000838.2006.00570.x

[11] Paine RM, Voight M. The role of the scapula. Journal of Orthopaedic \& Sports Physical Therapy; 1993; 18(1):386-91. doi: 10.2519/jospt.1993.18.1.386

[12] Barlow JC, Benjamin BW, Birt PJ, Hughes CJ. Shoulder strength and range-of-motion characteristics in bodybuilders. Journal of Strength and Conditioning Research. 2002; 16(3):367-72. doi: 10.1519/00124278-200208000-00006

[13] Leggin BG, Neuman RM, Iannotti JP, Williams GR, Thompson EC. Intrarater and interrater reliability of three isometric dynamometers in assessing shoulder strength. Journal of Shoulder and Elbow Surgery. 1996; 5(1):18-24. doi: 10.1016/ s1058-2746(96)80026-7

[14] Hurd WJ, Kaplan KM, Elattrache NS, Jobe FW, Morrey BF, Kaufman KR. A profile of glenohumeral internal and external rotation motion in the uninjured high school baseball pitcher, part II: Strength. Journal of Athletic Training. 2011; 46(3):289-95. doi: 10.4085/1062-6050-46.3.289

[15] Turner N, Ferguson K, Mobley BW, Riemann B, Davies G. Establishing normative data on scapulothoracic musculature using handheld dynamometry. Journal of Sport Rehabilitation. Human Kinetics; 2009; 18(4):502-20. doi: 10.1123/ jsr.18.4.502 
[16] Ekstrom RA. Comparing the function of the upper and lower parts of the serratus anterior muscle using surface electromyography. Journal of Orthopaedic and Sports Physical Therapy. 2004; doi: 10.2519/jospt.2004.1345

[17] Michener LA, Boardman ND, Pidcoe PE, Frith AM. Scapular muscle tests in subjects with shoulder pain and functional loss: Reliability and construct validity. Physical Therapy. 2005; doi: $10.1093 / \mathrm{ptj} / 85.11 .1128$

[18] Ekstrom RA, Soderberg GL, Donatelli RA. Normalization procedures using maximum voluntary isometric contractions for the serratus anterior and trapezius muscles during surface EMG analysis. Journal of Electromyography and Kinesiology. 2005; 15(4):418-28. doi: 10.1016/j.jelekin.2004.09.006

[19] Hislop H, Avers D, Brown M. Daniels and Worthingham's muscle testing: Techniques of manual examination and performance testing. Berlin: Elsevier Health Sciences; 2013.

[20] Ekstrom RA, Donatelli RA, Soderberg GL. Surface electromyographic analysis of exercises for the trapezius and serratus anterior muscles. Journal of Orthopaedic \& Sports Physical Therapy. 2003; 33(5):247-58. doi: 10.2519/ jospt.2003.33.5.247

[21] Smith J, Padgett DJ, Kaufman KR, Harrington SP, An K-N, Irby SE. Rhomboid muscle electromyography activity during 3 different manual muscle tests11No commercial party having a direct financial interest in the results of the research supporting this article has or will confer a benefit upon the author(s) or upon any organization with which the author(s) is/are associated. Archives of Physical Medicine and Rehabilitation. 2004; 85(6):987-92. doi: 10.1016/s00039993(03)00618-x

[22] Lorenz DS. The importance of the posterior capsule of the shoulder in overhead athletes. Strength and Conditioning Journal. 2005; 27(4):60-2. doi: 10.1519/00126548-20050800000010

[23] McClure PW, Michener LA, Karduna AR. Shoulder function and 3-dimensional scapular kinematics in people with and without shoulder impingement syndrome. Physical Therapy, 2006. 86(8):1075-1090. doi: 10.1093/ptj/86.8.1075

[24] Smith J, Dietrich CT, Kotajarvi BR, Kaufman KR. The effect of scapular protraction on isometric shoulder rotation strength in normal subjects. Journal of Shoulder and Elbow Surgery. 2006; 15(3):339-43. doi: 10.1016/j.jse.2005.08.023

[25] Alfredson H, Pietilä T, Lorentzon R. Concentric and eccentric shoulder and elbow muscle strength in female volleyball players and non-active females. Scandinavian Journal of Medicine \& Science in Sports. 1998; 8(5):265-70. doi: 10.1111/j.1600-0838.1998.tb00481.x

[26] Wilk KE, Meister K, Andrews JR. Current concepts in the rehabilitation of the overhead throwing athlete. The American Journal of Sports Medicine. 2002; 30(1):136-51. doi: $10.1177 / 03635465020300011201$

[27] Escamilla RF, Andrews JR. Shoulder muscle recruitment patterns and related biomechanics during upper extremity sports. Sports Medicine. 2009; 39(7):569-90. doi: 10.2165/00007256-200939070-00004

[28] Myers JB, Laudner KG, Pasquale MR, Bradley JP, Lephart $\mathrm{SM}$. Scapular position and orientation in throwing athletes.
The American Journal of Sports Medicine. 2005; 33(2):26371. doi: $10.1177 / 0363546504268138$

[29] Ganong WF, Ganong W. Review of medical physiology. Norwalk, CT: Appleton \& Lange Norwalk; 1995.

[30] Kisner C, Colby LA. Therapeutic exercise: foundations and techniques. Philadelphia: F. A. Davis Company; 2012.

[31] Donatelli R, Ellenbecker TS, Ekedahl SR, Wilkes JS, Kocher $\mathrm{K}$, Adam J. Assessment of shoulder strength in professional baseball pitchers. Journal of Orthopaedic \& Sports Physical Therapy. 2000; 30(9):544-51. doi: 10.2519/jospt.2000.30.9.544 